\title{
Clinical use of programmed cell death-1 (PD-1) and its ligand (PD- L1) expression as discriminatory and predictive markers in ovarian
}

\section{cancer}

Jayanta Chatterjee ${ }^{1 *}$, Wei Dai ${ }^{1}$, Nor Haslinda Abd Aziz ${ }^{1}$, Pei Yun Teo ${ }^{1}$, John Wahba ${ }^{1}$, David

L. Phelps ${ }^{1}$, Christian J. Maine ${ }^{1}$, Lynsey M. Whilding ${ }^{1}$, Roberto Dina ${ }^{1}$, Giorgia Trevisan ${ }^{1}$, Kirsty

J. Flower ${ }^{1}$, Andrew J. T. George ${ }^{1,2} \wedge$, Sadaf Ghaem-Maghami ${ }^{1 \wedge}$

* and ${ }^{\wedge}$ equal contribution from these authors

1. Imperial College London

2. Brunel University London

\section{Running Title}

PD-1 and PDL-1 as clinical markers in ovarian cancer

Keywords

PD1 PDL1 ovarian cancer biomarkers

\section{Financial Support}

JC received a fellowship from Imperial College Trust. NHAA received a PhD studentship from the Malaysian Ministry of Education. Funding was received from Mann-Hodgson charitable trust and Rosie's charity. 


\section{Corresponding author}

Dr Sadaf Ghaem-Maghami MRCOG PhD

Associate Professor and Honorary Consultant Gynaecological Oncology Surgeon

$4^{\text {th }}$ Floor IRDB, Hammersmith Hospital Campus, Du Cane Road, London W12 ONN

s.ghaem-maghami@imperial.ac.uk, +44 (0)20 33133267

\section{Conflicts of interest statement}

No authors declare any conflicts of interest

\section{Contributorship}

Conception and study design - AJTG, SGM

Data acquisition - CJM, JC, NHAA, JW, DLP, RD, GT, LMW

Data interpretation and analysis - all authors

Drafting of the manuscript - PYT, DLP, JC, WD, NHAA, KJF, SGM, AJTG

Critical appraisal and revision of manuscript - all authors

Abstract $247 / 250$ words max

Manuscript 3986/5000 words max 


\section{ABSTRACT}

\section{Purpose}

We aimed to establish whether PD-1 and PD-L1 expression, in ovarian cancer (OC) tumour tissue and blood, could be used as biomarkers for discrimination of tumour histology and prognosis of OC.

\section{Experimental Design}

Immune cells were separated from blood, ascites and tumour tissue obtained from women with suspected $O C$ and studied for the differential expression of possible immune biomarkers using flow cytometry. PD-L1 expression on tumour associated inflammatory cells was assessed by immunohistochemistry and tissue microarray. Plasma soluble PD-L1 was measured using sandwich ELISA. The relationships among immune markers were explored using hierarchical cluster analyses.

\section{Results}

Biomarkers from the discovery cohort that associated with PD-L1 ${ }^{+}$cells were found. PD-L1 ${ }^{+}$ $\mathrm{CD}_{14}{ }^{+}$cells and $\mathrm{PD}-\mathrm{L1}^{+} \mathrm{CD} 11 \mathrm{c}^{+}$cells in the monocyte gate showed a distinct expression pattern when comparing benign tumours and epithelial ovarian cancers (EOC) - confirmed in the validation cohort. Receiver operating characteristic curves showed PD-L1 ${ }^{+}$and PD-L1 ${ }^{+}$ CD14 ${ }^{+}$cells in the monocyte gate performed better than the well-established tumour marker CA-125 alone. Plasma soluble PD-L1 was elevated in EOC patients compared to healthy women and patients with benign ovarian tumours. Increased percentage of PD-L1 ${ }^{+}$ 
and PD-L1 ${ }^{+} \mathrm{CD} 14^{+}$monocytes and PD-1 $1^{+}$lymphocytes correlated with sub-optimal surgery. Low total PD-1 ${ }^{+}$expression on lymphocytes was associated with improved survival.

\section{Conclusions}

Differential expression of immunological markers relating to the PD-1/PD-L1 pathway in blood can be used as potential prognostic and diagnostic markers in EOC. These data have implications for the development and trial of anti PD-1/PD-L1 therapy in ovarian cancer.

\section{STATEMENT OF TRANSLATIONAL RELEVANCE}

This study is set apart from others in this field by using liquid biopsies to detect immune molecules of interest in the context of a solid tissue cancer. We found that differential expression of blood-based immune markers can discriminate benign tumours from ovarian cancer, appear to be prognostic and are associated with residual disease status after surgery. Furthermore our work shows that tissue-based immune markers are associated with survival and soluble PD-L1 in the blood may be a surrogate marker for expression of PD-L1 in tissue.

These are significant findings as these markers may be useful as clinical tools early in a patient's management by predicting prognosis and potential response to anti-PD- 1 therapy, which could be determined with a blood test. There is now an opportunity to target this pathway to achieve therapeutic response and improved survival using novel anti-PD-1 and anti-PD-L1 antibodies by the potential use of these biomarkers. 


\section{INTRODUCTION}

Ovarian cancer remains the most lethal gynaecological cancer in the developed world. Fiveyear survival is typically $46 \%{ }^{1}$ Advances in therapies have improved survival, but overall burden of mortality remains high, accounting for $4.2 \%$ of deaths worldwide. ${ }^{2}$ Standard treatment includes cytoreductive debulking surgery and chemotherapy. Epithelial ovarian cancer (EOC) accounts for $90 \%$ of all ovarian cancers and is therefore the focus of this research.

Patients who have no apparent residual disease following surgery (complete cytoreduction) have improved prognosis, however patients with EOC often present late which contributes to poor survival. ${ }^{3,4}$ No biomarkers currently exist to allow the accurate prediction of achieving complete cytoreduction, nor the patient's response to surgery. In women with a pelvic mass, an elevated CA- 125 has sensitivity of $72 \%$ and specificity of $78 \%$ for EOC. ${ }^{5}$ Only $50 \%$ of early-stage patients have increased CA-125. Therefore, CA-125 is an unreliable biomarker and the development of an accurate biomarker may improve diagnosis and possibly survival.

EOC is regarded as immunogenic and immune responses may play an important role in prognosis. For example, overall survival (OS) for EOC is influenced by the presence or absence of tumour infiltrating lymphocytes (TILs) (five-year OS; 73.9\% versus $11.9 \%$ respectively). ${ }^{6}$ The total number of lymphocytes has also been shown to be prognostic ${ }^{7}$ and the composition of tumour-infiltrating immune cells changes according to stage of disease. ${ }^{8}$ 
A meta-analysis evaluating the prognostic value of TILs in EOC has shown that intraepithelial $\mathrm{CD}^{+}$or $\mathrm{CD} 8^{+}$TILs correlates with significantly longer OS (hazard ratio (HR) 2.24, 95\% Cl 1.712.91; for TIL-negative tumours). ${ }^{9}$

The tumour microenvironment uses several mechanisms to evade anti-tumour immune responses. Programmed cell death ligand 1 (PD-L1) is highly up-regulated on some tumour cells as well as immune cells and has been implicated in tumour immune homeostasis. ${ }^{10-12}$ PD-L1 suppresses T cell activation when it interacts with its receptor (PD-1) expressed on activated T cells. ${ }^{13}$ Phosphorylation of the PD-1 receptor, upon ligand engagement, causes down regulation of the antigen receptor signalling and inhibits T cell proliferation.

High PD-L1 expression on tumour cells has been associated with poor prognosis in EOC. ${ }^{14}$ Furthermore, we have previously shown significant differences in the expression of PD-L1 on monocytes and PD-1 on T cells, obtained from blood and ascites, in patients with benign and malignant ovarian tumours. ${ }^{11}$ These studies demonstrate the potential for PD-1/PD-L1 to be used as diagnostic and prognostic markers in EOC. Targeted immunotherapies that block PD-1/PD-L1 interactions have shown great promise in the treatment of cancer and are being tested in EOC. ${ }^{15}$

In this study we investigate a panel of immunological markers, chosen based on previously published work in cancers including EOC, in order to identify diagnostic and prognostic markers that may facilitate clinical decision making. ${ }^{11,14,16}$ These markers may pave the way for a rapid test to discriminate between EOC and benign tumours. 


\section{PATIENTS AND METHODS}

Ethical approval was granted by West London \& GTAC Research Ethics Committee (04/Q0406/79 and 10/H0707/7).

Blood, ascites, and tumour samples were collected before or during surgery and before chemotherapy (174 women enrolled 2007-2012) (Supplementary Figure 1). Blood and/or ascites was obtained from 126 women: 11 healthy volunteers, 10 borderline ovarian tumours, 71 EOCs, and 34 benign ovarian tumours. Data from all patients with ovarian pathology (EOC, benign, borderline) were used in the creation of the heatmaps and data from healthy patients were used as a control group for the ELISA experiment. Data from benign and EOC patients were used to compile all other figures and the characteristics of these patients are summarised in Supplementary Table S1. The discovery set included 48 benign and EOC patients and the validation set 49. Data from 30 consecutive patient samples from the discovery set have been previously published. ${ }^{11}$ All EOC patients had primary cytoreductive surgery followed by standard chemotherapy; $72 \%$ achieved complete surgical cytoreduction. Tumour from a further 48 patients was analysed by immunohistochemistry (IHC) (33 high-stage serous EOC and 15 benign tumours).

Overall survival (OS) and progression free survival (PFS) were measured as days between diagnosis and death or progression respectively, or last known contact. Patients lost to follow-up were censored when they were last seen. Median follow-up was 20 months. CA125 was recorded at presentation. 


\section{Isolation of mononuclear cells from peripheral blood (PBMCs) and ascites (AMCs).}

Mononuclear cells were obtained from blood and ascites by gradient separation as previously published. ${ }^{11}$

\section{Flow cytometry}

Cells isolated from blood and ascites were incubated with Fc receptor blocking agent (Miletenyi Biotec, Surrey, UK). Mononuclear cells were stained with fluorescein isothiocyanate (FITC), phycoerythrin (PE) and allophycocyanin (APC) conjugated monoclonal antibodies against CD3, CD4, CD8, CD28, CD19, CD69, CD25, CD86, CD14, CD11c and PD-1 (BD Bioscience, Oxford, UK), FOXP3 (Biolegend, London, UK), CTLA-4 and HLA-DR (Abcam, Cambridge, UK) specific for surface and intracellular cell markers. Matched isotype controls were used for each antibody to determine the gates (Supplementary Figure 2). The full protocol has been previously published. ${ }^{11}$

\section{Construction of tissue microarrays and immunohistochemistry}

PD-L1 expression on tumour associated inflammatory cells was assessed by immunohistochemistry staining (Dako, Carpinteria, USA) sections of ovarian tumours. Tissue microarrays were constructed using 48 ovarian tumours. Each paraffin-embedded tissue block had two areas identified to represent the tumour's centre and invasive margin; five cores were taken from each tumour. Cores were cut $(5 \mu \mathrm{m})$ and then microwaved (10 min at $850 \mathrm{~W}$ in $\mathrm{pH} 6.0$ citrate buffer), for antigen retrieval, and stained with haematoxylin and eosin and for immunological markers using the avidin-biotin complex (ABC) (Vectorlab, Peterborough, UK) and Supersensitive Polymer detection (Biogenex, Freemont, USA). Total number and percentage of PD-L1 positive (PD-L1 ${ }^{+}$) tumour inflammatory cells was 
determined by two pathologists. The cells were identified by their morphology and location within the tumours i.e. epithelial or stromal. When multiple sections from one tumour were assessed, the mean was calculated.

\section{Hierarchical Clustering}

Hierarchical cluster analysis was applied using Euclidean distance and average linkage method. The stability of clusters was evaluated via a bootstrap resampling method implemented in pvclust package in R (v2.10.1), which estimated an approximately unbiased (AU) p value for each cluster using 1000 times multi-scale bootstrap resampling. ${ }^{17}$ Clusters were considered strongly supported by the data when the AU p value was $>95 \%$.

\section{Statistical Analysis}

Statistical analyses were performed using R and SPSS. Mann-Whitney $U$ test was used to assess differential expression of immune markers between tissue types (significance $p<0.05)$. In boxplots the upper and lower limits of the box represent $75^{\text {th }}$ and $25^{\text {th }}$ centiles respectively. The horizontal line represents median. Whiskers indicate upper or lower quartile plus or minus 1.5 times the interquartile range (IQR). Receiver operating characteristic (ROC) curves assessed each biomarker's tissue discriminatory ability by determining the area under the curve (AUC). OS and PFS was determined using univariate log-rank and multi-variable Cox proportional-hazards adjusting for age, stage, grade, histology and residual disease. EOC patients were allocated to groups (high or low) using the immunohistochemistry score's median expression level as the cut-off. Expression of markers was a continuous variable in Cox models. 


\section{Sandwich ELISA for the detection of soluble PD-L1}

Immuno Maxisorb 96-well plates (Fisher Scientific, Loughborough, UK) were coated with capture antibody (rabbit anti-human PD-L1, Sino-Biological, Beijing, China), diluted in coating buffer $(0.1 \mathrm{M}$ Sodium carbonate, $\mathrm{pH} 9.5)$ to a final concentration of $1 \mu \mathrm{g} / \mathrm{mL}$, overnight at $4^{\circ} \mathrm{C}$, then washed with wash buffer (PBS with $0.05 \%$ Tween-20). Assay diluent (AD) $(200 \mu \mathrm{l})$ (PBS with $10 \%$ heat inactivated FCS, at pH 7.0) was added as a blocking agent and incubated ( $1 \mathrm{~h}$ room temperature). Plasma samples were centrifuged at $22000 \mathrm{~g} .100 \mu \mathrm{l}$ of plasma samples or recombinant PD-L1 protein (Sino Biological), used to create the standard curve, were added to each well and incubated (overnight $4^{\circ} \mathrm{C}$ ). The plate was washed before $100 \mu \mathrm{l}$ of goat anti-human PD-L1 detection antibody (R\&D Systems, Abingdon, UK), $(0.1 \mu \mathrm{g} / \mathrm{ml})$, was added and incubated ( $2 \mathrm{~h}$ room temperature) and washed with wash buffer. Streptavidin-HRP complex (Biolegend, London, UK) was diluted 1:1000 in $\mathrm{AD}$ and added to the plate and incubated $(2 \mathrm{~h}$ room temperature). The plate was washed and $50 \mu$ of tetramethylbenzidine substrate (BD Bioscience, Oxford, UK) was added and incubated (30 min room temperature). Stop solution $\left(50 \mu \mathrm{l} 1 \mathrm{M} \mathrm{H}_{3} \mathrm{PO}_{4}\right.$ ) was added and absorbance at $450 \mathrm{~nm}$ measured. 


\section{RESULTS}

Discovery of differentially expressed immune markers in PBMCs and AMCs of patients with EOC or benign ovarian tumours.

To determine differential expression of immune markers between benign tumours and EOC, immune markers on PBMCs and AMCs were analysed by flow cytometry. Heatmaps represent immune marker data obtained from 48 patients in the discovery cohort tissue microarray (Figure 1). There were four distinct clusters of markers in PBMCs and AMCs (Supplementary Figure S3). Percentage of monocytes PD-L1 ${ }^{+}$, monocytes PD-L1 ${ }^{+} \mathrm{CD} 14^{+}$and monocytes $\mathrm{PD}-\mathrm{L}^{+}{ }^{+} \mathrm{CD} 11 \mathrm{c}^{+}$showed a distinct expression pattern when comparing benign tumours and EOCs (Figure 1A).

\section{Validation of discovered immune markers}

Some immune markers found in the monocyte gate were differentially expressed between benign tumours and EOC; percentage of PD-L1 ${ }^{+}$cells, PD-L1 ${ }^{+} \mathrm{CD} 14^{+}$cells, and PD-L1 ${ }^{+} \mathrm{CD} 11 \mathrm{c}^{+}$ cells (Figure 2A). Markers found in the lymphocyte gate also differed between the groups; percentage of $\mathrm{CD} 9^{+}$cells, $\mathrm{CD} 4^{+} \mathrm{PD}-1^{+}$cells and PD-1 ${ }^{+}$cells (Figure $2 \mathrm{~A}$ ). These results were consistent with the data obtained from the clustering analysis. To exclude the confounding effect of different histological subtypes and grade, we further confined the analysis to highgrade serous (HGS) and benign tumours. Differential expression remained significant in five of the originally discovered six immune markers (monocytes (PD-L1 $1^{+}, \mathrm{PD}-\mathrm{L1}^{+} \mathrm{CD} 14^{+}, \mathrm{PD}-\mathrm{L} 1^{+}$ $\left.\mathrm{CD} 11 \mathrm{c}^{+}\right)$and lymphocytes $\left(\mathrm{CD} 69^{+}\right.$and $\left.\left.\mathrm{CD} 4^{+} \mathrm{PD}-1^{+}\right)\right)$and marginal significance was observed in lymphocytes PD- $1^{+}$(Supplementary Figure S4A). Similar results were obtained for ascites (Supplementary Figure S5). 
Four of the immune markers from lymphocyte and monocyte subsets were chosen for validation (lymphocytes (PD- $1^{+}$and $\mathrm{CD} 69^{+}$), monocytes (PD- $\mathrm{L}^{+}$and $\left.\mathrm{PD}-\mathrm{L}^{+} \mathrm{CD} 14^{+}\right)$). Flow cytometry analysis was performed on blood obtained from a validation cohort of patients to confirm the previous results. Validation was only carried out in blood as this is more clinically usable as a diagnostic test than ascites. When the analysis was repeated in the validation cohort, all four immune markers continued to discriminate (Figure 2B and Supplementary Figure S4B).

Clinically it is important to distinguish between benign tumours and early stage EOC. To investigate differential expression between these tumours, PBMC expression of PD-L1 was analysed by flow cytometry. Stage I EOC had significantly elevated total PD-L1 ${ }^{+}$monocytes and PD-L1 ${ }^{+} \mathrm{CD} 14^{+}$cells in the monocyte gate compared to benign tumours (Figure $2 \mathrm{C}$ ). To assess the robustness of these markers at discriminating benign tumours and EOC, a receiver operating characteristic $(\mathrm{ROC})$ curve was created comparing total PD-L1 ${ }^{+}$ monocytes and PD- $1^{+}{ }^{+} \mathrm{CD} 14^{+}$cells in the monocyte gate to the well-established CA-125 biomarker (Figure 2D). Both PD-L1 $1^{+}$and $\mathrm{PD}-\mathrm{L1}^{+} \mathrm{CD} 14^{+}$cells in the monocyte gate were better predictors than CA-125 alone; PD-L1 ${ }^{+}$(AUC 0.94), PD-L1 ${ }^{+}$CD14 ${ }^{+}$cells (AUC 0.94), CA125 (AUC 0.91). PD-1 and CD69 expression on lymphocytes were not discriminatory (data not shown). To establish whether a combination of markers would be able to improve the discrimination of EOC from benign tumours we grouped PD-L1+ monocytes and PD1+ lymphocytes in the ROC analysis, which showed a comparable AUC of 0.93 as shown in supplementary figure S6A. Further ROC analysis of the high grade serous (HGS) sub-group revealed an AUC of 0.98 when using PD-L1+ monocytes alone and an AUC of 0.97 when combining PD-L1+ monocytes and PD1+ lymphocytes (Supplementary Figure S6B). 


\section{Investigation of soluble PD-L1 in plasma by enzyme-linked immunosorbent assay (ELISA)}

ELISA was used to determine soluble PD-L1 (SPD-L1) concentrations as this method is much quicker than flow cytometry and could potentially be developed into a test using SPD-L1 as a surrogate marker of tumour cell and immune cell surface expression of PD-L1. Soluble PD-L1 concentration in the plasma of healthy women, patients with benign tumours, and EOC was measured. Figure 3 shows that there were significantly higher levels of sPD-L1 in the plasma of EOC patients compared to healthy women and patients with benign tumours. There was no significant difference between SPD-L1 levels in patients with benign tumours and healthy individuals.

\section{Correlation of immune marker expression with surgical cytoreduction outcome}

To investigate any association between the four validated immune markers and surgical cytoreduction outcome, expression was compared in patients with different surgical outcomes. PD-L1 expression on monocytes and PD-1 expression on lymphocytes in PBMCs was significantly higher in patients who had sub-optimal surgery $(>1 \mathrm{~cm}$ residual disease) compared to those who had optimal surgery $(\leq 1 \mathrm{~cm}$ residual disease). Figure 4 shows that expression of three PBMC immune markers (\% monocytes PD-L1 ${ }^{+}, \%$ monocytes PD$\mathrm{L1}^{+} \mathrm{CD} 14^{+}, \%$ lymphocytes $\mathrm{PD} 1^{+}$) correlate with surgical outcome in the combined cohort (discovery and validation patients) while one, \% lymphocytes $\mathrm{CD}^{+} 9^{+}$, does not.

\section{Immune markers and association with survival}

The four validated immune markers (\% lymphocytes PD- $1^{+}, \%$ lymphocytes $C D 69^{+}, \%$ monocytes PD-L1 ${ }^{+}$and $\%$ monocytes PD-L1 ${ }^{+} \mathrm{CD} 14^{+}$) were analysed in PBMCs of EOC patients for the combined patient cohort $(n=63)$. Kaplan Meier survival curves and multivariable Cox 
models determined whether the immune markers were associated with PFS and OS. EOC patients were allocated to groups (high or low) using the PBMC immune marker's median expression level as the cut-off. Percentage of lymphocytes that were PD- $1^{+}$was associated with PFS (Cox $p=0.001$, adjusted HR 1.25, 95\% Cl 1.09-1.43) (Figure 5A and Supplementary Table S2). Low percentage of lymphocytes PD- $1^{+}$was associated with improved OS (Cox $\mathrm{p}=0.003$, adjusted HR 1.28, 95\% Cl 1.09-1.51) (Figure 5B and Supplementary Table S2). The other immune markers did not correlate with survival (Supplementary Figure S7).

\section{Validation of the significant immune markers by immunohistochemistry}

Immunohistochemistry was performed as a further validation of PD-L1 as a diagnostic marker in EOC. Consistent with the data obtained by flow cytometry, the results show a higher percentage of PD-L1 ${ }^{+}$tumour associated inflammatory cells in EOC when compared to benign tumour tissue (Figure 6A, B, C). PD-L1 expression on tumour associated inflammatory cells was analysed for prognostic value. Patients were dichotomised, into high and low PD-L1 immunohistochemistry expression score, using the median. A continuous Cox proportional hazards analysis showed, despite low sample numbers, that high percentage of PD-L1 ${ }^{+}$tumour associated inflammatory cells was associated with an increased risk of disease progression (Figure 6D). No association was found with overall survival. 


\section{DISCUSSION}

Current diagnostic models do not enable clinicians to reliably distinguish benign tumours from early-stage EOC. Therefore, there remains a clinical need for a robust diagnostic test for ovarian masses to ensure rapid recourse to the correct treatment for patients that require the most urgent intervention. Currently, clinical indices dictate whether EOC patients receive primary surgery or upfront chemotherapy, rather than tumour biology or a prognostic biomarker. The development of an accurate test to determine treatment and so improve outcome would be a significant step towards personalisation of care in ovarian cancer. Immunological biomarkers have useful prognostic potential in a variety of cancers $^{16,18}$ and can predict the response to novel immunotherapies such as anti-PD-L1 and anti-PD-1 blocking antibodies. ${ }^{19}$

PD-L1 expression on tumour has been correlated previously to prognosis. ${ }^{11,14,20}$ To our knowledge this is the first study investigating expression of immunological markers in the blood of women with ovarian masses. Our initial heatmap cluster analysis (Figure 1) identified strong clusters separating serous and endometrioid carcinomas from benign and borderline tumours - the clustering for mucinous and clear-cell tumours was less well defined. The numbers of mucinous and clear-cell patients precluded our ability to analyse these as sub-groups and therefore we combined them for all analyses as EOC. We would expect the cluster analysis to be more clear-cut if we had focussed on the serous and endometrioid subtypes, given their strong clustering in the heatmaps. However, we argue that it is important to include all EOC histology types together as one group in the analysis 
to reflect patient populations in the clinic, especially as all EOCs are clinically managed the same way with surgical cytoreduction and chemotherapy irrespective of histology.

We have shown differential PBMC expression of immune markers in patients with EOC and benign tumours in separate discovery and validation cohorts (Figure 2A, B). This is clinically valuable since PBMCs are easily obtained via relatively non-invasive blood tests. Metastasis of EOC is generally not thought to be haematogenous, therefore the discovery of immunological signatures in the blood is interesting and may represent a generalised systemic effect on the immune system with the up-regulation of PD-L1 and PD-1 representing an immunoregulatory response in EOC.

The difficulty in diagnosing EOC lies in differentiating stage I EOC from benign or borderline tumours. Our data suggests that PD-L1 expression on monocytes and CD14 ${ }^{+}$cells in blood is significantly up-regulated in stage I EOC compared to benign tumours (Figure $2 \mathrm{C}$ ) which may be a useful clinical tool in women who present with a pelvic mass. Stage I tumours are more likely to be clear-cell, mucinous or low-grade-serous. In the comparison between stage I EOC and benign tumours, endometrioid, HGS and mucinous subtypes accounted for $64 \%$, $21 \%$ and $15 \%$ of the tumours respectively. Therefore the differential expression of total PD$\mathrm{L1}^{+}$monocytes and PD-L1 ${ }^{+} \mathrm{CD} 14^{+}$mainly reflect the difference between stage I endometrioid and benign tumours. Due to small sample size in the mucinous and clear-cell subtypes, we were unable to perform the analysis in these two subtypes. Further investigation of these markers in different EOC subtypes and grades with adequate sample size would be warranted. 
Although advances have been made using predictive mathematical models and ultrasound, to distinguish ovarian tumour types, sensitivity can still be as low as $50 \%$ with specificity of $84 \% .{ }^{21}$ The positive predictive value of a single CA-125 measurement for the early detection of EOC is $57 \% .^{22}$ Sensitivity and specificity for the combination of the ultrasound algorithm and CA-125 in specialist centres can have an AUC of 0.94 (0.93-0.95) for discriminating allstage EOC from benign tumours. ${ }^{23}$ CA-125 in our cohort had a similar AUC of 0.91 (0.831.00); the wider AUC range likely due to smaller numbers in our cohort. We have shown that PD-L1 expression on circulating monocytes is comparable to CA-125 with an AUC of $0.94(0.89-0.98)$ for all cancer stages (Figure 2D). This marker therefore has the potential to serve as a useful tool for early stage EOC diagnosis. It appears from our results that little, if anything, is gained from combining multiple markers and PD-L1 ${ }^{+}$monocytes perform well as a single marker (Figure 2D and Supplementary Figure 6A, B). Sub-analysis of only HGS patients shows that PD-L1 ${ }^{+}$monocytes can be used as a biomarker to discriminate HGS from benign tumours (AUC 0.98) (Supplementary Figure 6B). However, the clinical dilemma is not the ability to discriminate HGS from benign disease, it is the ability to identify early stage EOC and therefore the analyses in figures $2 \mathrm{D}$ and supplementary figure $6 \mathrm{~A}$, which includes all histologies, would be more applicable to clinical practice.

Soluble PD-L1 in plasma of EOC patients was found to be significantly higher than in patients with benign tumours or healthy individuals (Figure 3). SPD-L1 has been shown to be elevated in the serum of patients with other cancers and was found to be associated with poor prognosis. ${ }^{24,25}$ It has also been shown that SPD-L1 is produced and released by activated mature dendritic cells and suggested that immune and tumour cells could be the source of soluble PD-L1. ${ }^{26}$ Measuring SPD-L1 may be a surrogate marker for surface PD-L1, 
which could be readily tested in the clinic with a blood test. Further work is required to explore whether these immunological markers in blood are raised in other conditions of inflammation, chronic infection and autoimmunity to establish their efficacy as diagnostic biomarkers and also to monitor response to therapy or recurrence of disease.

The volume of residual disease after surgery for EOC is strongly associated with survival. We have shown that percentage of lymphocytes PD-1 ${ }^{+}$, percentage of monocytes PD-L1 $1^{+}$, and percentage of monocytes PD-L1 ${ }^{+} \mathrm{CD} 14^{+}$in PBMCs significantly correlates inversely with surgical outcome (Figure 4). This is important, as accurately predicting complete resection of the tumour may have prognostic benefit. This could facilitate personalised treatment strategies especially as some studies have proposed that upfront neo-adjuvant chemotherapy with delayed surgery, in advanced EOC, seems to have similar prognosis to primary surgical debulking with adjuvant chemotherapy. ${ }^{27,28}$ Ideally we would have restricted this analysis to only advanced (stages III \& IV) patients, as these patients are most likely to achieve sub-optimal cytoreduction and have the worst prognosis. Due to relatively low numbers we included patients with all-stages of disease and therefore the effect seen in figure 4 may be as a result of the immune markers reflecting patients with high-stage disease. This would need to be further assessed using a more homogenous group including only high-stage patients.

Notably in our study, a low percentage of lymphocytes expressing PD-1 was associated with favourable survival outcome (Figures $5 \mathrm{~A}, 5 \mathrm{~B}$ and Supplementary Figure 7). This is a significant finding as it may be a useful prognostic tool, early in a patient's management. PD1 expression may also be of relevance in determining which patients may benefit from anti- 
PD-1/PD-L1 therapy. The median follow up in this study was 20 months. This relatively short period is a limitation in this prospective study. However, given that the median time to first recurrence is $18-24$ months, we can be confident in our estimation of progression free survival. Accurate determination of overall survival will require a longer follow up.

Immunohistochemical analysis of tumour tissue has shown that tumour associated inflammatory cells had different PD-L1 expression in benign tumours and EOC (Figure 6A, B, C). Furthermore, the PD-L1 expression on tumour associated inflammatory cells appears to be significantly associated with prognosis, specifically PFS (Figure 6D). Previous studies have reported correlation with prognosis and PD-L1 expression on tumour cells across different histological subtypes of EOC. ${ }^{14,20}$ Our data is based on PD-L1 expression on tumour associated inflammatory cells, rather than the tumour cells themselves. This may have more biological significance from an immunological standpoint as a recent study, based on tissue samples of patients treated with therapeutic anti-PD-L1 antibody, showed that response correlated to the presence of PD-L1 positive tumour associated macrophages. ${ }^{19}$

We have shown that increased expression in monocytes of PD-L1 ${ }^{+}$and PD-L1 ${ }^{+} \mathrm{CD} 14^{+}$cells and expression in lymphocytes of $\mathrm{PD}-1^{+}$and $\mathrm{CD}^{+} 9^{+}$cells is associated with $\mathrm{EOC}$, and that expression of PD-1 on lymphocytes correlates with prognosis. These markers may therefore be useful as diagnostic and/or prognostic tools in this disease. Furthermore, given the importance of immunoregulation through PD-1 and PD-L1, this suggests that expression of PD-1 and PD-L1 on monocytes and lymphocytes may provide important information on how and when blocking antibodies to these molecules may work. 


\section{ACKNOWLEDGEMENTS}

Thanks to Imperial College Healthcare NHS Trust patients who provided samples. Support

was provided by Experimental Cancer Medicine Centre, National Institute for Health Research Biomedical Research Centre at Imperial College London and Imperial College Healthcare Trust. 


\section{REFERENCES}

1. CRUK. Ovarian cancer survival 2014. http://www.cancerresearchuk.org/health-professional/cancer-statistics/statistics-bycancer-type/ovarian-cancer/survival\#heading-Zero (accessed 18/11/2016).

2. Ferlay J, Shin HR, Bray F, Forman D, Mathers C, Parkin DM. Estimates of worldwide burden of cancer in 2008: GLOBOCAN 2008. Int J Cancer 2010; 127(12): 2893-917.

3. Elattar A, Bryant A, Winter-Roach BA, Hatem M, Naik R. Optimal primary surgical treatment for advanced epithelial ovarian cancer. Cochrane Database Syst Rev 2011; (8): Cd007565.

4. Chang SJ, Hodeib M, Chang J, Bristow RE. Survival impact of complete cytoreduction to no gross residual disease for advanced-stage ovarian cancer: a meta-analysis. Gynecol Oncol 2013; 130(3): 493-8.

5. Patsner B, Mann WJ, Chalas E. Predictive value of CA 125 for ovarian carcinoma in patients presenting with pelvic masses. Obstet Gynecol 1988; 71(6 Pt 1): 949-50.

6. Zhang L, Conejo-Garcia JR, Katsaros D, et al. Intratumoral $\mathrm{T}$ cells, recurrence, and survival in epithelial ovarian cancer. $N$ Engl J Med 2003; 348(3): 203-13.

7. Milne $\mathrm{K}$, Alexander $\mathrm{C}$, Webb JR, et al. Absolute lymphocyte count is associated with survival in ovarian cancer independent of tumor-infiltrating lymphocytes. J Transl Med 2012; 10: 33.

8. Fialova A, Partlova S, Sojka L, et al. Dynamics of T-cell infiltration during the course of ovarian cancer: the gradual shift from a Th17 effector cell response to a predominant infiltration by regulatory T-cells. Int J Cancer 2013; 132(5): 1070-9.

9. Hwang W-T, Adams SF, Tahirovic E, Hagemann IS, Coukos G. Prognostic significance of tumor-infiltrating T cells in ovarian cancer: A meta-analysis. Gynecologic Oncology 2012; 124(2): 192-8.

10. Dong H, Strome SE, Salomao DR, et al. Tumor-associated B7-H1 promotes T-cell apoptosis: a potential mechanism of immune evasion. Nat Med 2002; 8(8): 793-800.

11. Maine CJ, Aziz NH, Chatterjee J, et al. Programmed death ligand-1 over-expression correlates with malignancy and contributes to immune regulation in ovarian cancer. Cancer Immunol Immunother 2014; 63(3): 215-24.

12. Curiel TJ, Wei S, Dong H, et al. Blockade of B7-H1 improves myeloid dendritic cellmediated antitumor immunity. Nat Med 2003; 9(5): 562-7.

13. Keir ME, Butte MJ, Freeman GJ, Sharpe AH. PD-1 and its ligands in tolerance and immunity. Annu Rev Immunol 2008; 26: 677-704.

14. Hamanishi J, Mandai M, Abiko K, et al. The comprehensive assessment of local immune status of ovarian cancer by the clustering of multiple immune factors. Clin Immunol 2011; 141(3): 338-47.

15. Brahmer JR, Tykodi SS, Chow LQ, et al. Safety and activity of anti-PD-L1 antibody in patients with advanced cancer. $N$ Engl J Med 2012; 366(26): 2455-65.

16. Galon J, Mlecnik B, Bindea G, et al. Towards the introduction of the 'Immunoscore' in the classification of malignant tumours. J Pathol 2014; 232(2): 199-209.

17. Shimodaira H. Approximately Unbiased Tests of Regions Using Multistep-Multiscale Bootstrap Resampling. The Annals of Statistics 2004; 32(6): 2616-41.

18. Galon J, Costes A, Sanchez-Cabo F, et al. Type, density, and location of immune cells within human colorectal tumors predict clinical outcome. Science 2006; 313(5795): 1960-4.

19. Herbst RS, Soria JC, Kowanetz M, et al. Predictive correlates of response to the anti-PDL1 antibody MPDL3280A in cancer patients. Nature 2014; 515(7528): 563-7.

20. Hamanishi J, Mandai M, Iwasaki M, et al. Programmed cell death 1 ligand 1 and tumorinfiltrating CD8+ T lymphocytes are prognostic factors of human ovarian cancer. Proc Natl Acad Sci U S A 2007; 104(9): 3360-5. 
21. Timmerman D, Ameye L, Fischerova D, et al. Simple ultrasound rules to distinguish between benign and malignant adnexal masses before surgery: prospective validation by IOTA group; 2010.

22. Kobayashi E, Ueda Y, Matsuzaki S, et al. Biomarkers for screening, diagnosis, and monitoring of ovarian cancer. Cancer Epidemiol Biomarkers Prev 2012; 21(11): 1902-12.

23. Van Calster B, Van Hoorde K, Valentin L, et al. Evaluating the risk of ovarian cancer before surgery using the ADNEX model to differentiate between benign, borderline, early and advanced stage invasive, and secondary metastatic tumours: prospective multicentre diagnostic study. Bmj 2014; 349: g5920.

24. Frigola X, Inman BA, Lohse CM, et al. Identification of a soluble form of B7-H1 that retains immunosuppressive activity and is associated with aggressive renal cell carcinoma. Clin Cancer Res 2011; 17(7): 1915-23.

25. Zheng $\mathrm{Z}, \mathrm{Bu} \mathrm{Z}$, Liu X, et al. Level of circulating PD-L1 expression in patients with advanced gastric cancer and its clinical implications. Chin J Cancer Res 2014; 26(1): 10411.

26. Frigola X, Inman BA, Krco CJ, et al. Soluble B7-H1: differences in production between dendritic cells and T cells. Immunol Lett 2012; 142(1-2): 78-82.

27. Vergote I, du Bois A, Amant F, Heitz F, Leunen K, Harter P. Neoadjuvant chemotherapy in advanced ovarian cancer: On what do we agree and disagree? Gynecol Oncol 2013; 128(1): 6-11.

28. Morrison J, Haldar K, Kehoe S, Lawrie TA. Chemotherapy versus surgery for initial treatment in advanced ovarian epithelial cancer. Cochrane Database Syst Rev 2012; 8: CD005343. 


\section{FIGURE LEGENDS}

Figure1

Heatmaps of candidate biomarkers in blood and ascites.

In PBMC samples 23 markers were analysed (A) and 22 markers were studied in ascites samples (B). Unsupervised hierarchical clustering of candidate biomarkers in healthy controls $(\mathrm{HH})$, benign ovarian tumours $(\mathrm{BN})$, borderline ovarian tumour $(\mathrm{BT})$ and EOCs (SER: serous; END: endometrioid; CC: clear cell; MU: mucinous).

* cluster is well supported by data (AU p>95\%), + cluster not significant (AU p value $90 \%$ )

Figure 2

Differential expression of immune markers on PBMCs in patients with benign tumours and EOCs in the discovery and validation cohorts.

A: The percentage of monocytes and lymphocytes (and their subsets) in PBMCs from patients with benign tumours or EOC that expressed PD-1, PD-L1 and CD69. B: Differential expression of PBMC markers between benign tumours and EOC in the validation set. Analysis was repeated after excluding data $>4$ standard deviations (SD) from the mean, to ensure statistical significance was not driven by extreme outliers, and all results remained significant $\left(p=0.0019, \%\right.$ monocytes PD-L1 ${ }^{+} / p=0.0004, \%$ monocytes PD-L1 ${ }^{+} C D 14^{+}, p=0.009$, $\%$ lymphocytes PD- $1^{+} / \mathrm{p}=0.026, \%$ lymphocytes $\mathrm{PD}-1^{+}$) C: The percentage of monocytes in PBMCs that were PD-L1 ${ }^{+}$or PD- $\mathrm{L}^{+} \mathrm{CD} 14^{+}$in the validation cohort was significantly different in patients with benign ovarian tumours and stage I EOC. Analysis was repeated after excluding data $>4$ SD from the mean to ensure statistical significance was not driven by extreme outliers. Both results remained significant $(p=0.005, \%$ monocytes PD-L1+ / 
$\mathrm{p}=0.005, \%$ monocytes $\left.\mathrm{PD}-\mathrm{L}^{+} \mathrm{CD} 14^{+}\right)$. Statistical analysis was performed using the MannWhitney $U$ test $(A-C)$. D: Receiver operating characteristic curve (combined cohorts $n=63$ ) showing the sensitivity and specificity of PD-L1 ${ }^{+}$and $\mathrm{PD}-\mathrm{L}^{+}{ }^{+} \mathrm{CD} 14^{+}$cells, compared to CA125, at discriminating EOC from benign tumours; AUC (95\% Cl): PD-L1 ${ }^{+}=0.94(0.89-0.98)$, PD-L1 ${ }^{+}$CD14 ${ }^{+}$cells $=0.94(0.90-0.98)$, CA-125 $=0.91(0.83-1.00)$.

Figure 3

Expression of SPD-L1 in plasma from healthy females and women with benign and EOC tumours.

The SPD-L1 was detected using ELISA. Bars indicate the mean expression. Statistical analysis was performed using the Mann Whitney U test.

Figure 4

Box plots showing surgical cytoreduction outcome and percentage of cells expressing the validated PBMC immune markers in patients with EOC.

Increased percentage of monocytes that are PD-L1 ${ }^{+}$or PD-L1 $1^{+} \mathrm{CD} 14^{+}$and lymphocytes that are PD $-1^{+}$strongly correlated with sub-optimal surgical outcome. Statistical analysis was performed using the Mann-Whitney $U$ test.

Figure 5

Kaplan-Meier plots of progression free survival (A) and overall survival (B) according to expression of PD-1 in lymphocytes. 
Expression of PD-1 on lymphocytes in blood obtained by flow cytometry $(n=63)$. Log-rank $p=0.023(A)$ and $p=0.095(B)$. The hazard ratio $(H R)$ was estimated by multivariable Cox models adjusted for age, stage, grade, histology, and residual disease.

Figure 6

Immunohistochemical tissue staining of PD-L1 on tumour associated inflammatory cells.

A: Moderate score staining of PD-L1 ${ }^{+}$inflammatory cells in EOC. B: Low score staining of PD$\mathrm{L}^{+}$inflammatory cells in benign ovarian tumour tissue. C: Percentage of PD-L1 ${ }^{+}$ inflammatory cells in benign and EOC tissue; statistical analysis was performed using the Mann-Whitney U test. D: Kaplan Meier survival curve showing significantly improved PFS in women with EOC who have above median (high) levels of PD-L1 ${ }^{+}$inflammatory cells in tumour tissue (log-rank $\mathrm{p}=0.024)$. Cox model adjusted for age, stage, grade, histology and residual disease. 

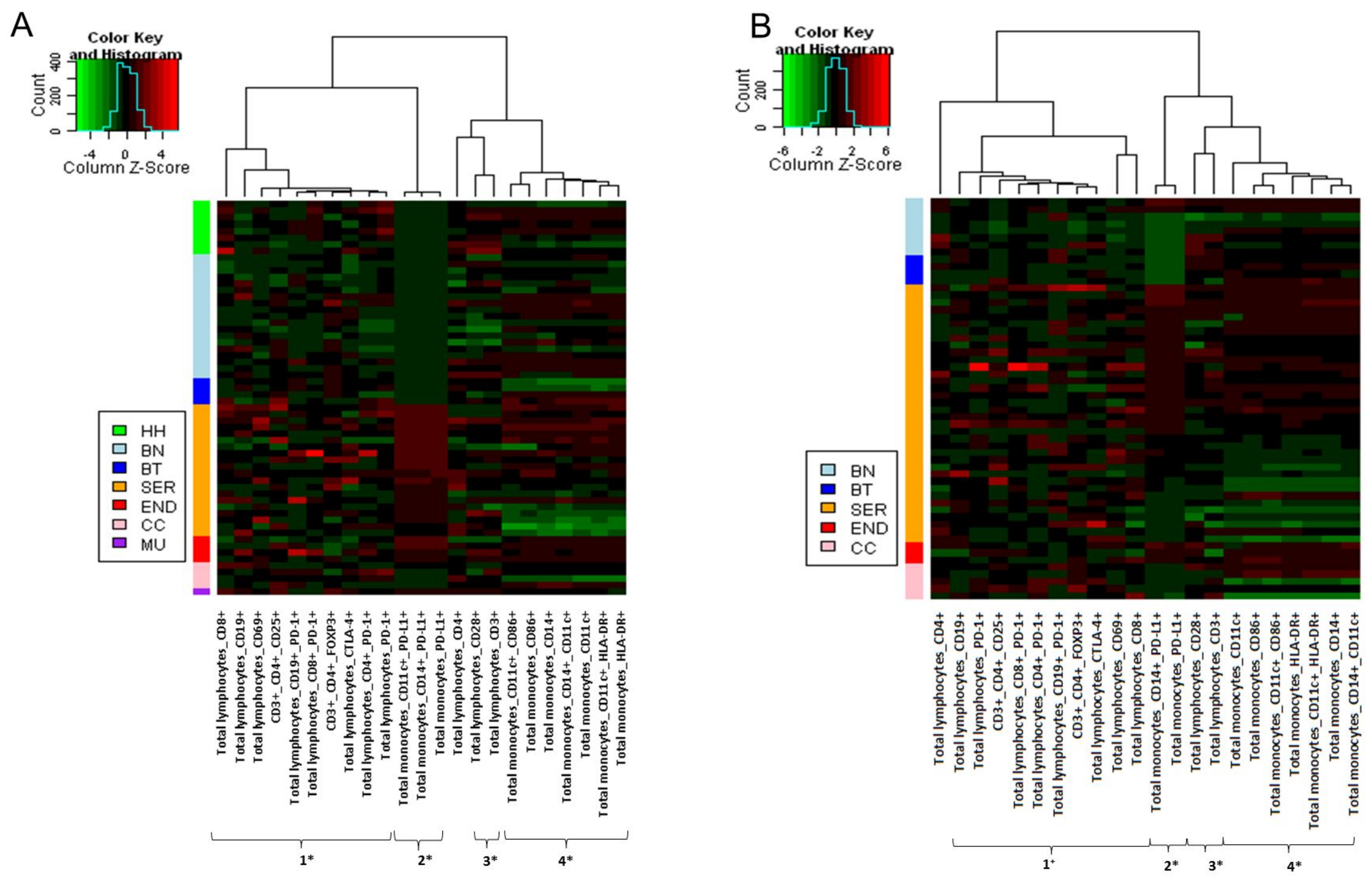
A
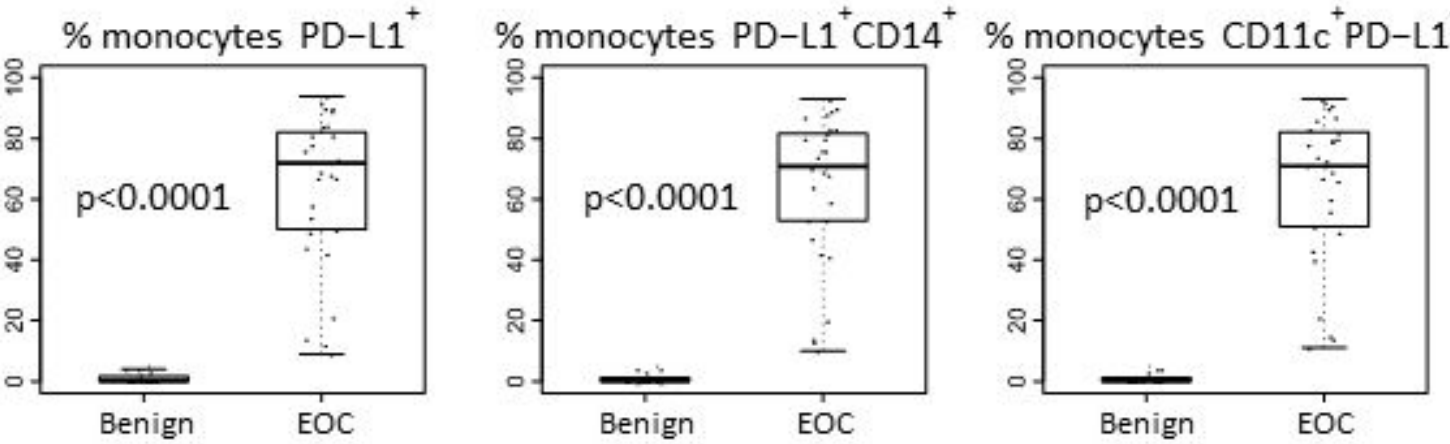

\% lymphocytes $\mathrm{CD} 69^{+}$

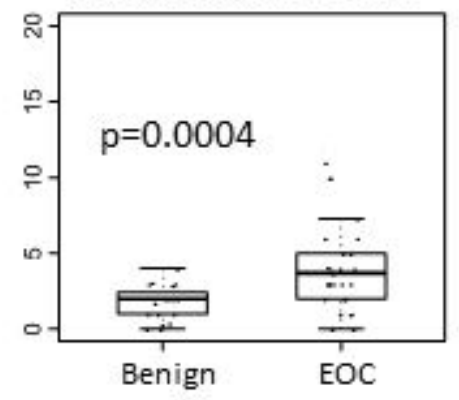

\% lymphocytes PD1 ${ }^{+}$

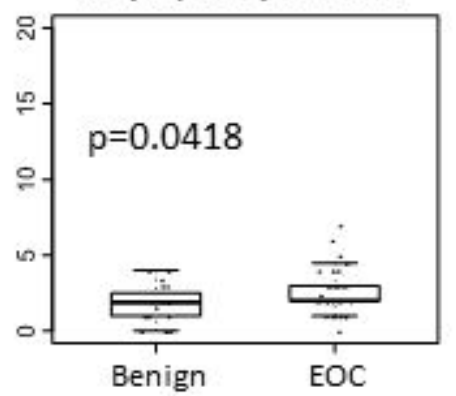

$\%$ lymphocytes $\mathrm{CD} 4{ }^{+} \mathrm{PD} 1^{+}$

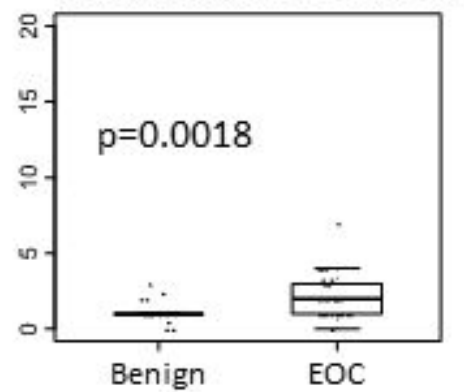

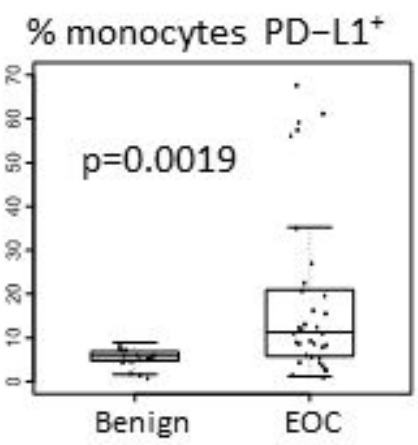

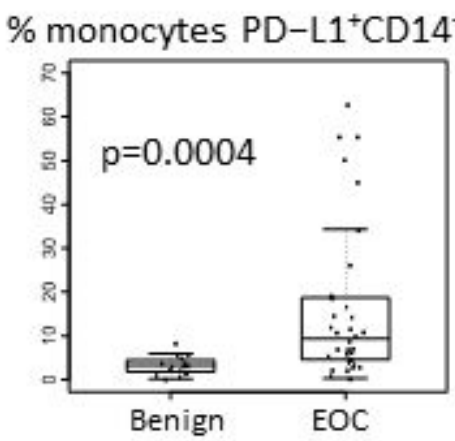

\% lymphocytes PD1 ${ }^{+}$

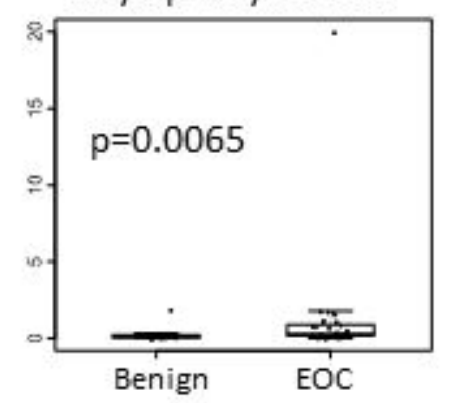

\% lymphocytes $\mathrm{CD}_{69}{ }^{+}$



D

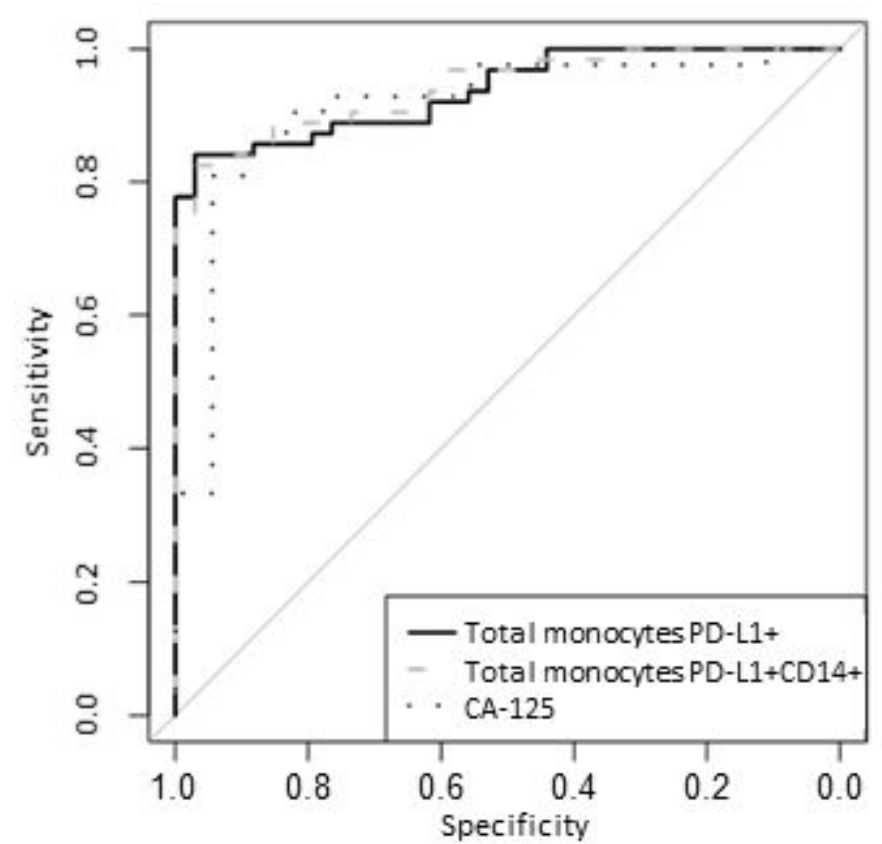

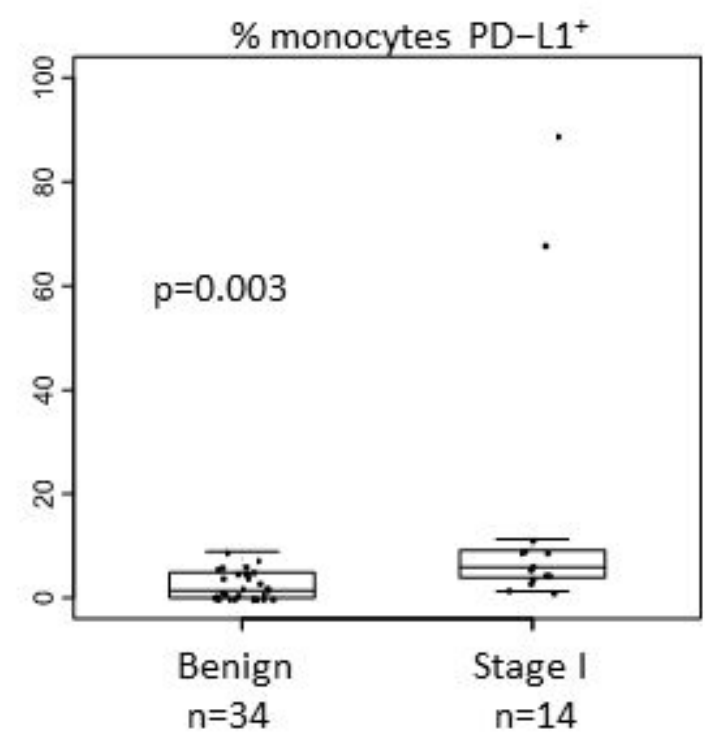

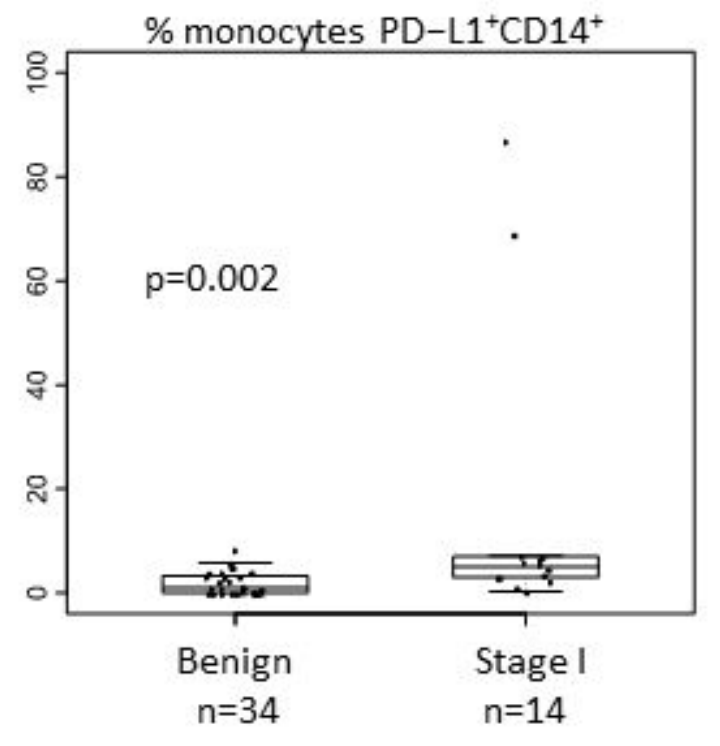


$p<0.0001$

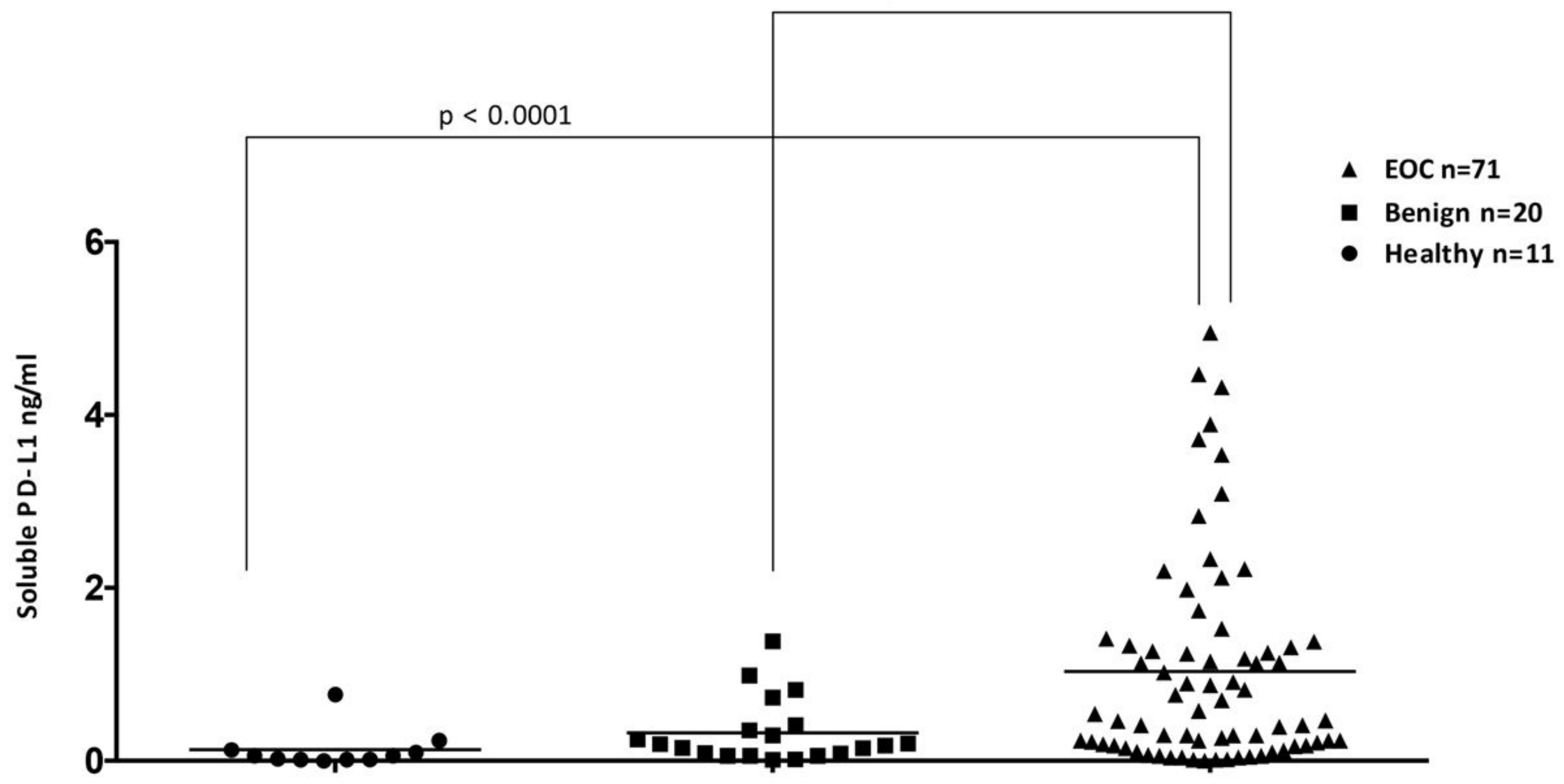


\% monocytes PD-L1+

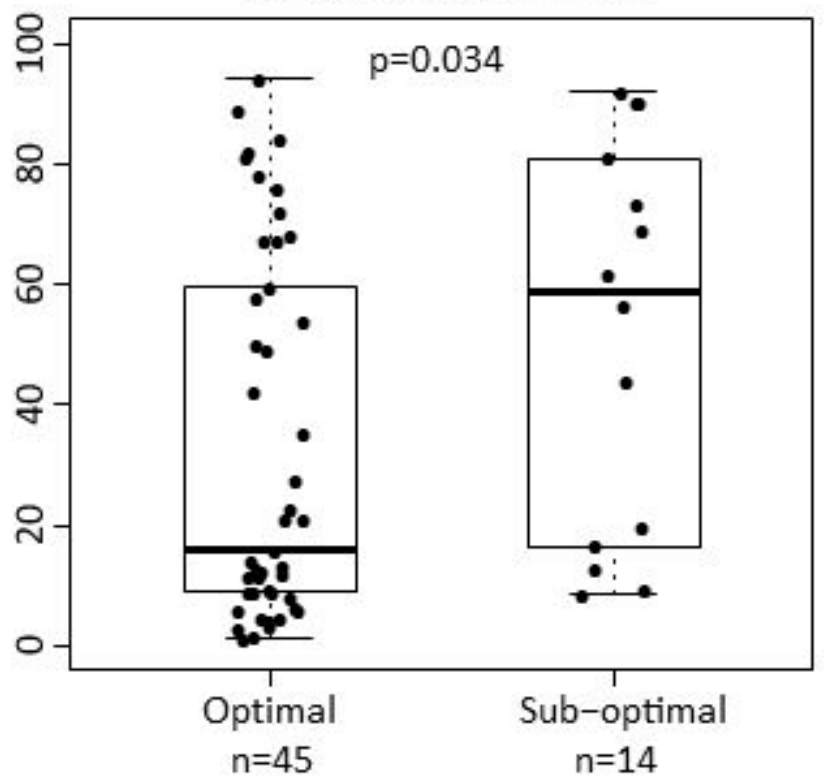

\% lymphocytes PD1 ${ }^{+}$

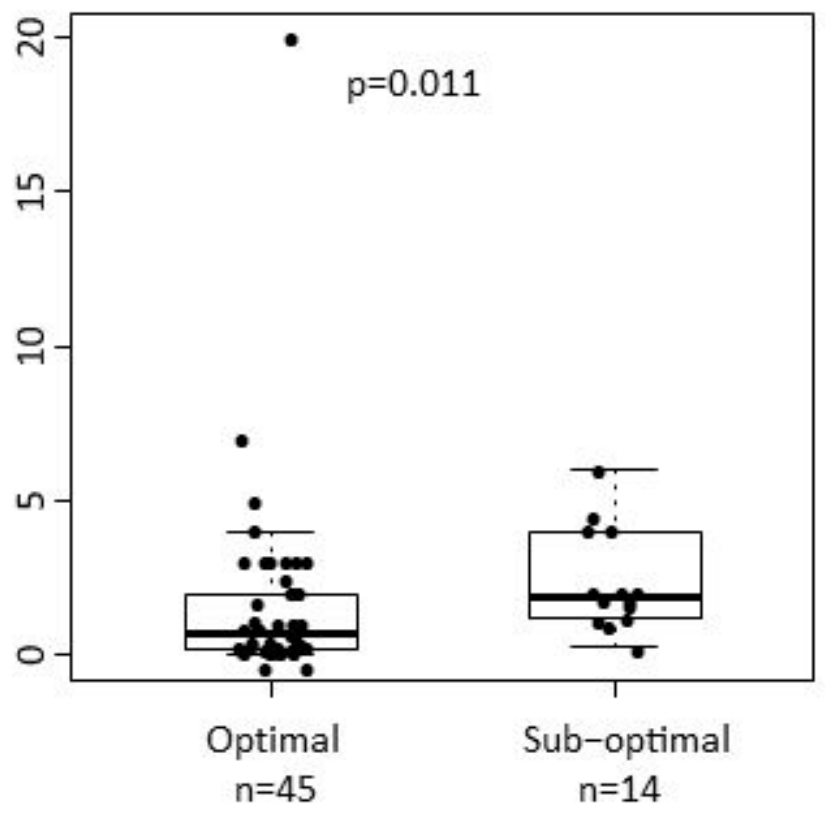

$\%$ monocytes $\mathrm{PD}-\mathrm{L} 1^{+} \mathrm{CD} 14^{+}$



\% lymphocytes CD69+






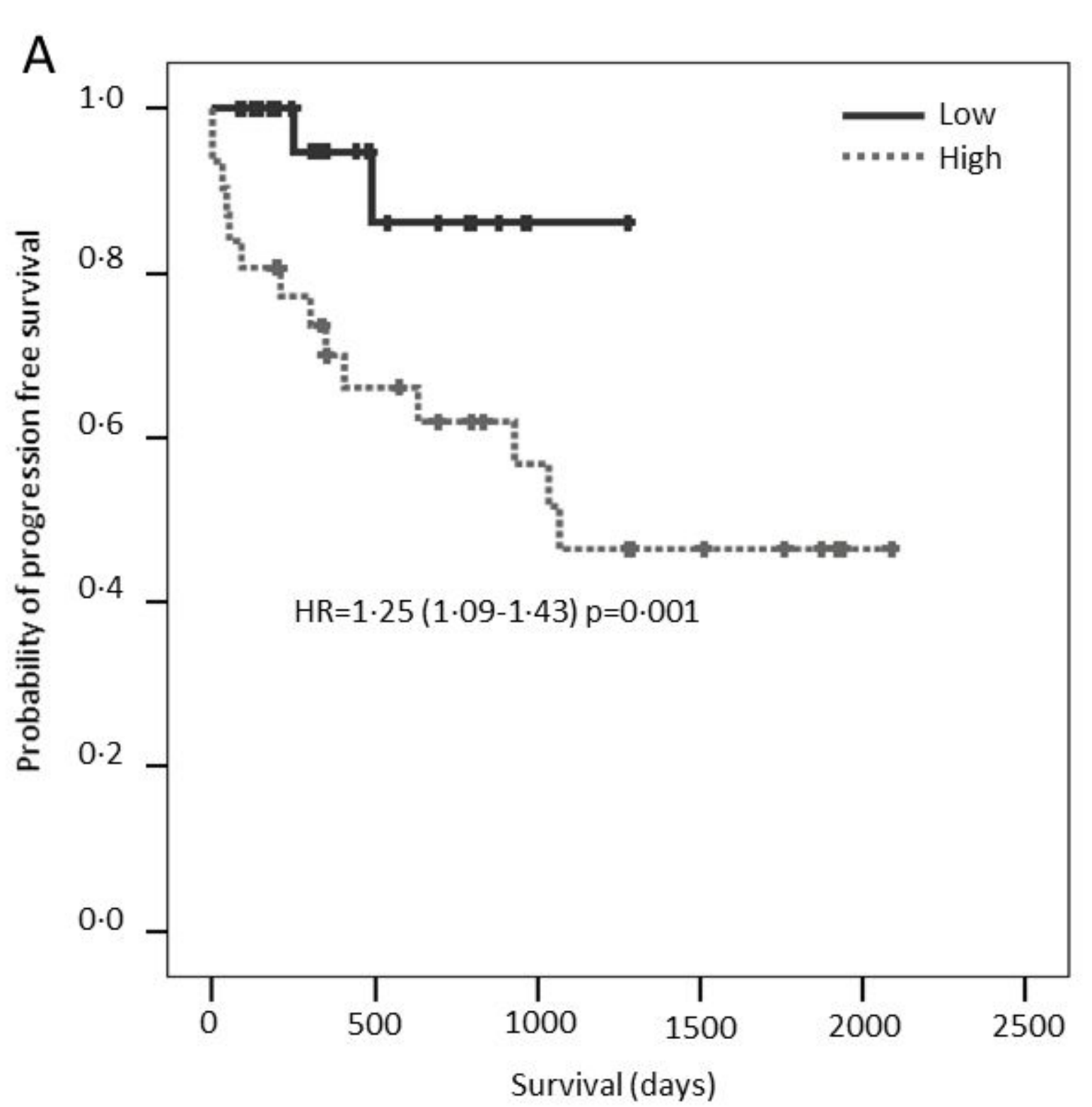

B

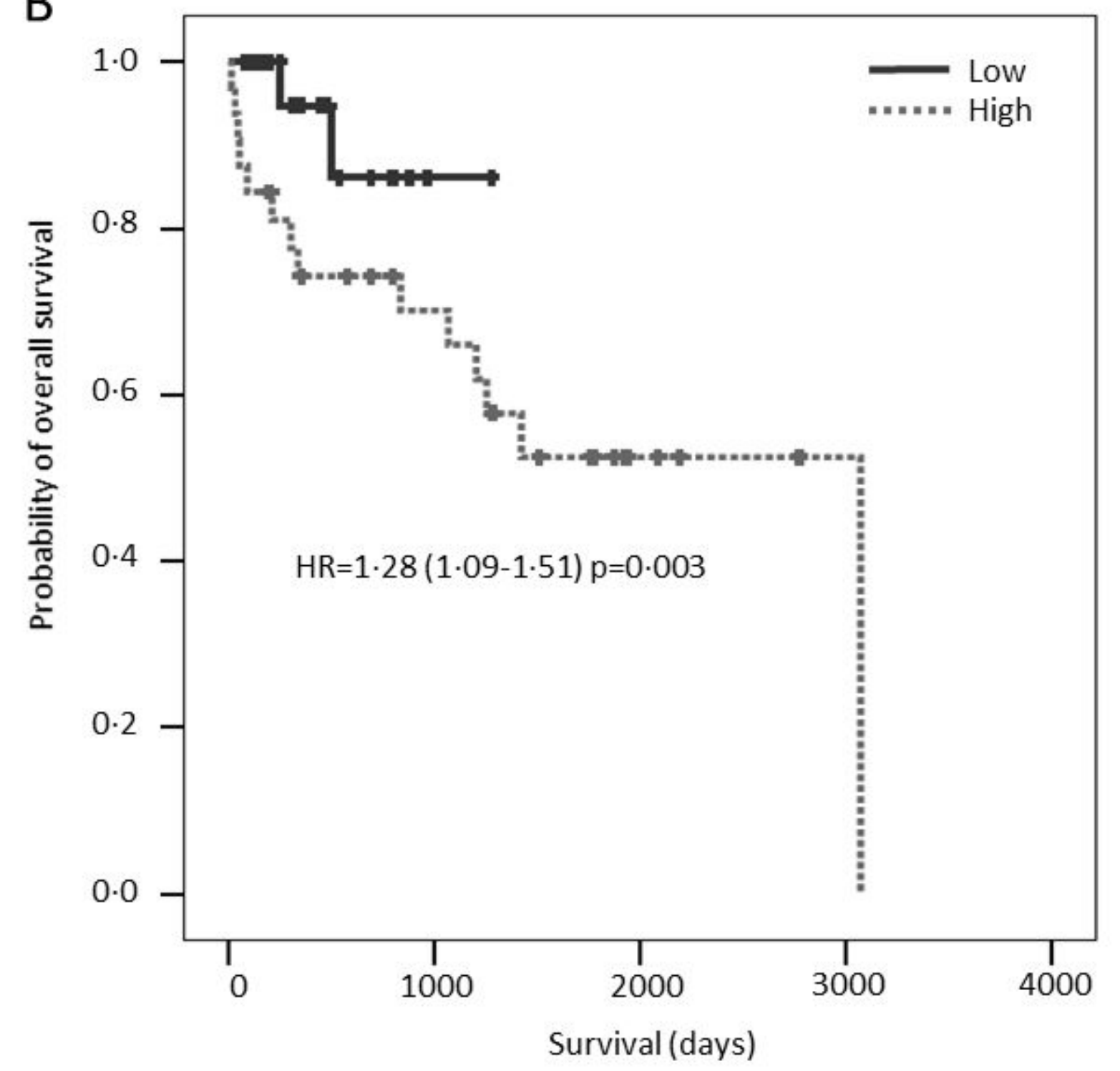


A

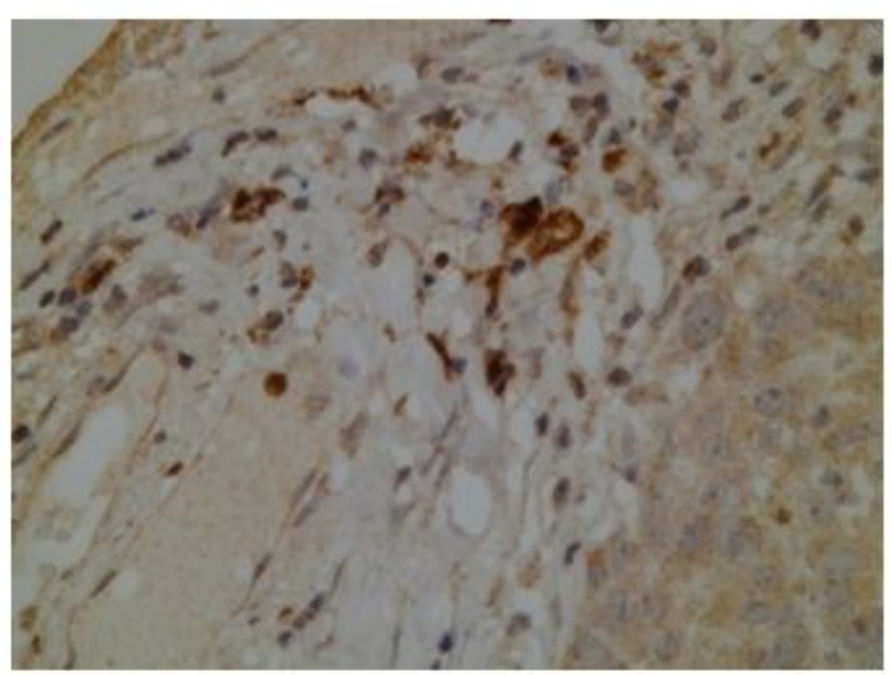

C



B

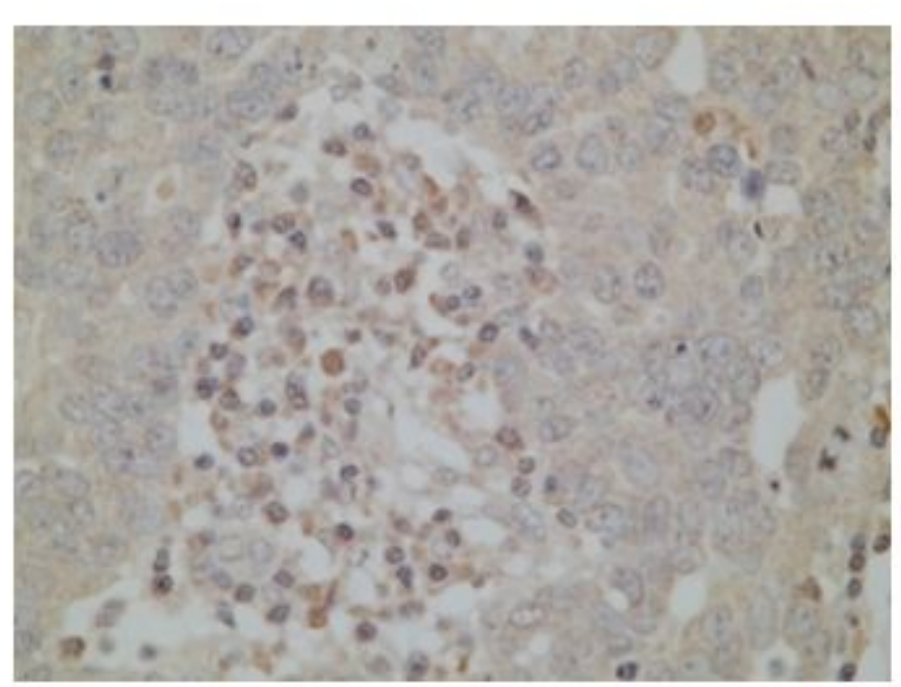

D



